\title{
La rehabilitación de la persona después de Wittgenstein y Apel, en Anscombe y Llano. ¿A través de Aristóteles o de nuevo Frege?
}

\author{
The rehabilitation of the person \\ after Wittgenstein and Apel, \\ in Anscombre and Llano. \\ Through of Aristotle or Frege again?
}

\author{
CARLOS ORTIZ DE LANDÁZURI. \\ Universidad de Navarra
}

Recibido: 29/11/2018 Aceptado: 03/03/2019

\begin{abstract}
RESUMEN
Anscombe y Llano pretendieron definir el constitutivo formal de la persona a partir de las propuestas de Wittgenstein o de éste conjuntamente con Apel. Sin embargo para ello primero tuvieron que evitar las paradojas que se originaron entonces, ya sea recuperando la noción de sujeto intérprete del correspondiente silogismo práctico aristotélico, ya sea revisando el uso que Wittgenstein y Apel habían hecho de sus respectivas teorías fregeanas del significado. En cualquier caso Anscombe definiría a la persona como un «signo interprete» intencional de ella misma, mientras que Llano añadiría que se trata específicamente de un «signo formal» de naturaleza intencional.
\end{abstract}

\section{PALABRAS CLAVE}

INTENCIONALIDAD, SILOGISMO PRÁCTICO, TEORÍA DEL SIGNIFICADO, SIGNO FORMAL.

(C) Contrastes. Revista Internacional de Filosofía, vol. XXIV-N² (2019), pp. 43-59. ISSN: 1136-4076 Departamento de Filosofía, Universidad de Málaga, Facultad de Filosofía y Letras Campus de Teatinos, E-29071 Málaga (España) 


\begin{abstract}
Anscombe and Llano tried to define the formal constitutive of the person based on the proposals of Wittgenstein or of him jointly with Apel. However, they first had to avoid the paradoxes that originated then, either by recovering the notion of the interpreting subject from the corresponding Aristotelian syllogism, or by revising the use that Wittgenstein and Apel had made of their respective Fregean theories of meaning. In any case Anscombe would define the person as an intentional «sign interpreter» of herself, while Llano would add that it is specifically a «formal sign» of an intentional nature.
\end{abstract}

KEYWORDS

INTENCIONALITY, PRACTICAL SYLLOGISM, THEORY OF MEANING, FORMAL SING.

\title{
I. LA PERSONA COMO SIGNO INTERPRETE INTENCIONAL EN ANSCOMBE.
}

MARgaret Anscombe en Intentions ${ }^{1}$ (1957) habría recurrido al silogismo práctico aristotélico para caracterizar el constitutivo formal de la persona. Pero a su vez también habría tratado de evitar las tres paradojas que se hicieron presentes en las dos épocas de Wittgenstein - como ahora sucede con el solipsismo metodológico, con la paradoja del lenguaje privado y con la del lenguaje meramente conductista. En cualquier caso Wittgenstein no habría podido alcanzar una correcta caracterización del correspondiente sujeto intérprete de un conjunto de «convenciones tácitas enormemente complejas» que permiten relacionar los signos y las diferentes intencionalidades de los participantes en un dialogo racional. A este respecto afirma Anscombe: «Aquí tenemos un caso de «convenciones tácitas enormemente complejas» que acompañan a nuestra comprensión del lenguaje común, como dijo Wittgenstein en el Tractatus. [...] «La descripción de una acción humana es enormemente compleja, si uno tuviera que decir qué interviene verdaderamente en ella ... y no obstante, incluso un niño puede dar ese informe» [...] El «razonamiento práctico» de Aristóteles o mi serie de preguntas «¿Por qué?» puede contemplarse como un mecanismo que revela el orden que existe en este caos». ${ }^{2}$

De todos modos ahora se hacen notar los mecanismos de orden mental mediante los que dicho sujeto intérprete relacional podría justificar su peculiar modo de apropiarse del correspondiente mundo entorno, o de interactuar con los respectivos interlocutores interpelados. De este modo pasaría a concebirse como un sujeto relacional verdaderamente personal que sería capaz de justificar y de hacerse responsable de la peculiar configuración que otorga a su respectivo mundo entorno. Para ello sujeto intérprete personal debe comenzar planteán-

1 Anscombe, E. Intentions, Blackwell, Oxford, 1957; Intención, Paidós, Barcelona, 1991.

2 Anscombe, Intentions, Ibidem, $140 \mathrm{p}$. 
dose la pregunta acerca del «porqué» y de las razones de los hechos positivos efectivamente acontecidos, por tratarse de un rasgo diferencial que caracteriza al constitutivo formal de la persona. Sin embargo, al tratar de contestar este tipo de cuestiones, el sujeto intérprete personal primeramente debe tratar de hacer coincidir el juego epistémico experimental del «ver» mediante el que comprueba el acaecer de unos determinados «hechos», con el juego semiótico compartido del «ver con los ojos de la mente», mediante el que comprueba su adecuada comprensión es esos mismos hechos. Solo así la descripción de los hechos acaecidos por recurso a un razonamiento práctico aristotélico se puede formular en el mismo juego de lenguaje que el usado para justificar las respectivas razones para creer en estos mimos hechos. A este respecto afirma Anscombe: «La conclusión es que el juego lingüístico de «ver» forma parte necesaria del juego de «ver con los ojos de la mente»; o más bien, que sólo podemos identificar al último como un juego lingüístico si el primero se construye con las mismas palabras». ${ }^{3}$

En este contexto Anscombe trata de reconstruir la profunda unidad existente entre las dos épocas Wittgenstein, el periodo positivista lógico y su posterior filosofía de los juegos del lenguaje. Por su parte, también tratará de mostrar la intrínseca articulación existente entre dos tipos de intencionalidad o de predicción justificada, a saber: por un lado, las razones para actuar, que están basadas en una posible predicción intencional de hechos positivos de carácter privado, pero referidos un modo crítico transcendental a toda la humanidad, tal y como habría propuesto en el solipsismo metodológico del Tractatus $;{ }^{4} \mathrm{y}$, por otro lado, las razones para creer en la veracidad de esos mismos hechos positivos en virtud de un juego del lenguaje discursivo de carácter conductista, dada la imposibilidad de lograrlo a través de un juego de lenguaje privado, como el mismo mostró en las Investigaciones. De este modo se localizan unos hechos de carácter solipsista cuya ocurrencia se justifica en virtud de unas razones para actuar de carácter compartido, haciendo que los hechos abandonen su inicial carácter solipsista. A este respecto afirma Anscombe: «En un principio, al considerar las expresiones de intención, he señalado que se trataba de predicciones justificadas, en todo caso en razones para actuar, como opuestas a la razones para creer en su veracidad. De modo que ya aquí he distinguido un sentido de «¿Por qué»?, en el cual la respuesta menciona hechos. «Habrá

3 Anscombe, E., Intentions, Ibidem, 73 p.

4 Wittgenstein, L., Tractatus Logico-Philosophicus, Routledge, 1922; Alianza, Madrid,1937 (1 $\left.1^{\mathrm{a}}\right), 1973\left(2^{\mathrm{a}}\right)$. 
un eclipse de luna». «¿Por qué?». «Porque ...», y la respuesta constituye una razón para creer que así será». ${ }^{5}$

Además, esta articulación presenta una circunstancia ahora añadida, a saber: en el caso de no poder justificar el acontecer de este tipo de hechos positivos por recurso a un silogismo práctico, en ese caso tampoco se podrían legitimar las correspondientes razones para creer en nombre de un juego del lenguaje discursivo conductista. De este modo no solo la noción de «sujeto intérprete» personal, sino también la propia noción de causalidad, se encontrarían en ese selecto grupo de las cuestiones que no pueden ser conocidas directamente por observación y, por tanto, tampoco se podrían legitimar mediante un juego de lenguaje discursivo de naturaleza conductista. A este respecto afirma Anscombe: «Una respuesta a la pregunta «¿Por qué?» que no ofrezca razones para creer en la realidad del hecho no proporciona, por lo tanto, una razón para actuar [...] Ahora bien, podemos ver que los casos en que se presenta esta dificultad son precisamente aquellos en que la causa misma qua causa (o tal vez deberíamos decir la causalidad misma) se encuentra en la clases de cosas conocidas sin observación». ${ }^{6}$

De todos modos Anscombe opina que mediante el silogismo práctico aristotélico se podrán resolver este tipo de dificultades: primero, permitirá lograr una descripción de los hechos positivos acontecidos que evite el dogmatismo crítico-transcendental y el solipsismo metodológico que se hizo presente en el Tractatus. En efecto, en la misma medida que se dispone de unas razones para creer que pueden ser compartidas por el conjunto de los interlocutores interpelados, tampoco estos hechos positivos deberían solo poder configurar un «mi mundo» o un «tu mundo» de carácter interpretativo dogmático y solipsista. Segundo, se requerirá inquirir unas razones para creer que permitan a su vez evitar el carácter incomunicable del lenguaje privado o de un lenguaje discursivo meramente conductista, como ocurrió en las Investigaciones. En efecto, en la medida que se dispone de unos hechos positivos que se remiten de un modo efectivo al respectivo mundo entorno circundante, tampoco las respectivas razones para creer deberían adolecer de un carácter semiótico incomunicable o meramente convencional. En cualquier caso Anscombe opina que una prolongación de las propuestas de Wittgenstein siguiendo la metodología del razonamiento práctico Aristotélico permitiría localizar el auténtico constitutivo formal del sujeto intérprete personal, sin dejarse llevar por posturas solipsistas, herméticas o simplemente conductistas A este respecto afirma Anscombe: «Existe una diferencia de forma entre el razonamiento que conduce a la acción

5 Wittgenstein, L., Philosophical Investigations, Blackwell, Londres, 1958; Investigaciones Filosóficas, Crítica, Barcelona, 1988. Cf. Anscombe, M., Intentions, Ibidem, 59 p.

6 Anscombe, M., Intentions, Ibidem, 59 p. 
y el razonamiento para encontrar la verdad de una conclusión. [...] Y existe el silogismo práctico propiamente dicho. Aquí la conclusión es una acción cuyo sentido es mostrado por las premisas, las cuales, ahora, están, por así decir, en servicio activo. Cuando Aristóteles afirma que lo que ocurre es lo mismo, parece querer decir que siempre se trata del mismo mecanismo psíquico por medio del cual una conclusión es educida»». ${ }^{7}$

Pero a pesar de este tipo de críticas, Anscombe opina que Wittgenstein siguió admitiendo la posibilidad de justificar el constitutivo formal del sujeto intérprete personal desde posturas que no fueran crítico-transcendentales, ni tampoco defensoras de un lenguaje privado en sí mismo incomunicable o de un lenguaje discursivo meramente conductista. En este sentido opina que son claras las referencias de Wittgenstein a la posibilidad de «abrir la intimidad» o de «compartir las intenciones del propio obrar» por parte del supuesto sujeto intérprete relacional respecto a un posible interlocutor. Además, habría admitido la posibilidad de compartir las intenciones, ya sea respecto de los hechos acontecidos o de las correspondientes razones para creer en la veracidad de unos mismos hechos. Al menos así sucede cuando se trata de justificar lo que se «iba a hacer» en una determinada circunstancia. En esos casos se pretende comunicar una intención que pretende ir más allá de los hechos acontecidos, aunque sin tampoco poder prescindir de ellos, ya que permiten legitimar las razones para creer en aquello que se pretendía hacer. En cualquier caso Wittgenstein no habría llevado a cabo a lo largo de sus dos épocas un ejercicio de autoobservación sobre las intenciones y motivaciones que le habrían terminado moviendo a lo largo de los diversos periodos de su vida, como ahora hace notar Anscombe. En su lugar habría llevado a cabo más bien un análisis de los requisitos incondicionados que se deben exigir a todo obrar intencional de carácter práctico. A este respecto afirma Anscombe: «También aquí Wittgenstein dice algo pertinente, en su discusión relativa a «iba a hacer»: «¿Por qué quiero, además de manifestar lo que hice, también comunicarte una intención? ... Porque quiero comunicarle algo sobre mí, algo que va más allá de lo que entonces ocurrió. // Le abro mi intimidad cuando le digo lo que querría hacer: - Pero no según una autoobservación, sino mediante una reacción» (Investigaciones filosóficas, \& 659). Posiblemente Wittgenstein piensa en una respuesta, o reacción, ante el recuerdo de «ese tiempo»; en el contexto que nos interesa, podemos pensarla como una respuesta a nuestra pregunta especial (sobre la intencionalidad de una acción)». ${ }^{8}$

En cualquier caso la realización de una acción intencional puede tener diversos presupuestos crítico-transcendentales o recurrir a distintas formas de

7 (Anscombe, M., Intentions, Ibidem, $114 \mathrm{p}$.

8 Anscombe, M., Intentions, Ibidem, $95 \mathrm{p}$. 
lenguaje discursivo meramente conductista. Sin embargo la intención última con la que el sujeto intérprete personal realiza una determinada acción intencional sólo la conoce el mismo. En efecto, por más que se pretenda justificar una correspondencia entre los hechos acontecidos y las razones para creer en esos mismos hechos, al final la intención con que se realiza algo sólo se conoce en la profunda intimidad de uno mismo. Especialmente cuando se comprueba que siempre es posible introducir una ruptura entre aquella doble dimensión crítico-transcendental y semiótico-discursiva respecto de una acción intencional, sin que la realización de los hechos acontecidos responda necesariamente a los motivos alegados. Sin embargo no se trata de una ruptura que tenga que venir generada por la influencia de condicionantes externos, que también puede haberlos, sino que se trata fundamentalmente de decisiones que han sido tomadas en la más estricta intimidad de uno mismo. En este caso la única prueba posible que se puede aportar es la confesión de las intenciones del propio sujeto intérprete personal cuando analiza la génesis de sus propias acciones. De todos modos ello no impide que también puedan existir otros signos indicio más o menos evidentes que permitan corroborar o desmentir esta misma declaración de intenciones por parte de un posible interlocutor ajeno. A este respecto afirma Anscombe: «Finalmente, sólo uno sabe si esa es su intención o no». [...] En otras palabras, «sabe» significa aquí simplemente «puede decir», a menos que imaginemos un caso en el cual se pueda afirmar: pensó que esta era su intención, pero resultó evidente que se engañaba». ${ }^{9}$

En cualquier caso el razonamiento especulativo-práctico ahora se concibe como un peculiar «camino» heurístico o indagador mediante el que sujeto intérprete personal reconoce su propia intimidad frente a otras posibles experiencias ajenas que también pueden remitirse de un modo crítico-transcendental o semiótico discursivo al respectivo mundo entorno y a otros posibles interlocutores. De este modo la acción intencional se concibe preferentemente como un proceso o camino heurístico mediante el que se alcanza un conocimiento de la propia intimidad personal, a través del ejercicio de la propia acción práctica. De ahí que el conocimiento de la persona y de la propia intimidad sea resultado de un conocimiento práctico, más que estrictamente teórico. A este respecto afirma Anscombe: «La «acción intencional» siempre presupone lo que podría denominarse «conocer el camino propio con respecto a» los asuntos incluidos en la descripción (completa) bajo la cual una acción puede llamarse intencional, y este conocimiento se ejerce en la acción y es práctico». ${ }^{10}$

Evidentemente el ejercicio práctico de la intencionalidad requiere del ejercicio de un mecanismo heurístico o «causa mental» personal, que justifique el

9 Anscombe, M., Intentions, Ibidem, 98-99 pp.

10 Anscombe, M., Intentions, Ibidem, 151 p.

Contrastes vol. XXIV-N² (2019) 
respectivo «porqué». Es decir, la localización de una razón por la que se cree en la validez de esos mismos hechos acontecidos, ya sean llevados a cabo por uno mismo o por alguien ajeno. En cualquier caso el ejercicio práctico de dicha «causa mental» personal nos permite alcanzar un reconocimiento reflexivo de uno mismo al tener que hacerse responsable de aquello que se ha hecho. Sin embargo la «causa mental» personal se puede referir no solo a las acciones, sino también a las sensaciones o incluso a los propios pensamientos. Lo decisivo en estos casos es separar las causas y los motivos, de modo que el simple conocimiento intencional de un objeto puede causar la correspondiente reacción de miedo, enfado o temor, sin que necesariamente este tenga que ser su auténtico motivo. En efecto, como hizo notar Wittgenstein, habitualmente este tipo de reacciones ya no se pueden justificar mediante criterios crítico-transcendentales o semióticos discursivos. En su lugar se deben justificar mediante un motivo más profundo, que solo se puede conocer desde la propia intimidad de cada persona. A este respecto afirma Anscombe: «Al considerar las acciones es importante distinguir entre causas mentales y motivos: al considerar sentimientos, como miedo o enfado, es importante distinguir entre causas mentales y objetos del sentimiento (...). El objeto del temor puede ser la causa del temor, pero, como comenta Wittgenstein, no es en cuanto tal la causa del temor». ${ }^{11}$

De todos modos no siempre el propio protagonista de una acción es el que mejor puede conocer los motivos de su particular modo de obrar. En efecto, dada la multitud de descripciones que siempre caben hacer de una acción, puede ocurrir que se preste atención a una de ellas, dejando a otras desatendidas. De este modo una persona puede creer que está haciendo $\mathrm{X}$, cuando se identifica con esa intención. Sin embargo esa misma persona puede decir que no estaba haciendo $X$, sin que haya que cuestionar la veracidad de lo que dice, dado que simplemente podría no estar prestando atención a determinados aspectos de su actuación, a pesar de que podían formar parte de la realización de X. De ahí que lo decisivo sea la intencionalidad que se determina en cada caso desde la propia intimidad personal. A este respecto afirma Anscombe: «Al considerar las acciones es importante distinguir entre causas mentales y motivos: al considerar sentimientos, como miedo o enfado, es importante distinguir entre causas mentales y objetos del sentimiento (...). El objeto del temor puede ser la causa del temor, pero, como comenta Wittgenstein, no es en cuanto tal la causa del temor». ${ }^{12}$

En cualquier caso si se desea investigar la intencionalidad de una acción se debe otorgar una prioridad a la causalidad mental personal, aunque posteriormente esa misma intención también puede materializarse en la realización

11 Anscombe, M., Intentions, Ibidem, 59-60 pp.

12 Anscombe, M., Intentions, Ibidem 54-55 pp. 
de una acción. De ahí que los criterios críticos transcendentales o semióticodiscursivos de la realización de una acción tengan un carácter muy secundario. Es más, se podría decir que el análisis de los hechos acontecidos tiene un interés muy secundario, cuando lo prioritario es la intención que se decide desde la propia intimidad personal. A este respecto afirma Anscombe: «Si queremos comprender qué es la intención tendremos que investigar una materia cuya existencia se da puramente en el ámbito de la mente, y aún cuando la intención desemboca en acciones, y la forma en que esto sucede presenta problemas interesantes, lo que ocurre físicamente, es decir, lo que esta persona hace en realidad, es lo último que necesitamos considerar en nuestra investigación. Aunque quisiera afirmar que es lo primero». ${ }^{13}$

Sólo así se podrán analizar las diversas dimensiones del obrar intencional en toda su complejidad, estableciendo las consiguientes relaciones de prioridad o subsidiariedad que en cada caso convengan. De igual modo que también se podrán llevar a cabo el consiguiente análisis factorial más especializado de algunas de las otras dimensiones más específicas del obrar intencional y no intencional por separado. En cualquier caso el razonamiento especulativopráctico aporta el mecanismo heurístico o causa mental personal más básica a la que se remite cualquier actuación en nombre propio, a saber: el propio actuar intencional como constitutivo formal específico de la persona. A este respecto afirma Anscombe: «Podemos decir simplemente: «Observe a ese hombre y explique qué está haciendo» [...] En la mayoría de los casos, lo que puede decirse es lo mismo que el hombre sabe, y también en la mayoría de ellos, aunque no en todos, se estará informando de una de las intenciones, a saber: la realización de una acción. Es más, si esa no fuera la intención, casi siempre quedará claro aún sin preguntárselo».. ${ }^{14}$

Según Anscombe, el último Wittgenstein también habría compartido una noción semejante de obrar intencional en nombre propio, sin dejarse llevar exclusivamente por las propuestas de tipo critico-transcendental o semiótico-público. De todos modos siempre puede ocurrir que las apreciaciones de una determinada persona caigan en una flagrante contradicción, por atribuir un excesivo protagonismo a este otro tipo de factores. Por ejemplo, cuando se pretende negar la intencionalidad de una acción en nombre propio alegando razones de necesidad crítico-transcendental o de simple conveniencia semiótico-convencional, siendo así que dicha acción se concibe a su vez como un medio necesario para alcanzar un determinado fin. Se genera así una contradicción performativa donde el rechazo de la propia autoría de dicha acción intencional permite confirmar su efectiva ejecución por parte de dicho

13 Anscombe, M., Intentions, Ibidem, $51 \mathrm{p}$.

14 Anscombe, M.; Intentions, Ibidem, $50 \mathrm{p}$.

Contrastes vol. XXIV-N² (2019) 
sujeto intérprete personal, a pesar de sus intentos por desmentirlo. Pero salvo esta excepción, que permite confirmar la regla, siempre hay que otorgar una prioridad a la declaración de intenciones por las que la persona se concibe a sí misma como un sujeto epistémico y como un signo intérprete de sus propios fines y virtualidades. Al menos este fue el modo utilizado por el último Wittgenstein a la hora de valorar la efectiva buena o mala conducta con que materialmente se llevó a cabo una determinada acción, cuando lo prioritario hubiera sido analizar el propósito con que se hizo, como el mismo hace notar. De este modo Wittgenstein habría tratado de mostrar como el sentimiento de vergüenza se acrecienta cuando se pretende negar la autoría de una determinada acción, cuando, con independencia de su contenido, el mismo debería haber sido el primero en reconocerla. A este respecto Anscombe afirma: «Podemos encontrar casos en que «solo la persona puede decir si tenía cierta intención o no», (....) y esto sirve para explicar lo que dijo Wittgenstein en el \& 644 de Investigaciones filosóficas: «No me avergüenzo de lo que hice entonces, sino del propósito que tenía. ¿Y no estaba el propósito también en lo que hice? ¿Qué justifica la vergüenza? La historia entera del incidente». ${ }^{15}$

En cualquier caso el obrar intencional en nombre propio define en Anscombe a la persona, en la medida que se concibe como un sujeto epistémico y como un signo intérprete de sí misma. Solo así la persona puede ser capaz de apropiarse del ejercicio de la intencionalidad cuando actúa en nombre propio. En efecto, solo concibiéndose como un sujeto epistémico y como un signo interprete de ella misma puede la persona seguir apropiándose de su conducta vital, sin hacerla depender de causas ajenas. Es más, en estos casos también se debe presuponer una relación epistémica previa respecto de los distintos objetos de la experiencia y de su correspondiente mundo entorno, por tratarse de un requisito para poder considerarse como un signo intérprete de uno mismo. A este respecto afirma Anscombe: «Esto demuestra una vez más que no es posible tomar ningún proceso (ni siquiera interior) como un acto de intención en sí mismo, pues si describimos un proceso, el hecho de que haya tenido lugar no constituye una prueba de intención»». ${ }^{16}$

En cualquier caso se puede decir que en todos estos supuestos la persona se concibe a sí misma como un sujeto y un signo intérprete con la intención de «hacer lo que en cada caso hace». Especialmente cuando se comprueba como la persona se define como un signo intencional de modo que se le puede asignar una triple dimensión especulativo-práctico-vital a su modo de actuar, a saber: la realización de su propio proyecto vital en su totalidad; la comisión de una determinada acción concreta en un determinado mundo entorno; o, finalmente,

15 Anscombe, M., Intentions, Ibidem, $95 \mathrm{p}$.

16 Anscombe, M., Intentions, Ibidem, $100 \mathrm{p}$. 
la atribución de una naturaleza racional que le permite hacerse responsable de la realización de este tipo de procesos. Posteriormente von Wright y Peter Winch también habrían concebido a la persona como un signo intérprete intencional de ella misma. Por su parte, Kripke y Strawson habrían propuesto una articulación de la triple dimensión icónica, indexical y performativa o realizativa de los actos de habla en el marco del correspondiente mundo entorno. A este respecto Anscombe afirma: «En términos generales, las personas tienen la intención de hacer lo que hacen». [...] Por ello es absurda la tesis que en ocasiones se sostiene: la acción intencional de una persona solo puede referirse mediante la descripción de su objetivo»». ${ }^{17}$ Pero veamos que afirma Llano a este respecto.

\section{II.- LA PERSONA COMO SIGNO FORMAL RELACIONAL EN LLANO.}

Alejandro Llano en «Metafísica y lenguaje» ${ }^{18}$ (1983) habría recurrido a la teoría del significado de Frege para justificar el constitutivo formal de la persona, procurando evitar a su vez las paradojas que se hicieron presentes en Wittgenstein o más tarde en Apel. ${ }^{19}$ A este respecto Llano discrepa del papel que Apel otorga a la máxima pragmática de Peirce a la hora de articular el sujeto intérprete relacional, con el mundo entorno y con un posible interlocutor, como si de este modo se pudieran prolongar a su vez las propuestas del segundo Wittgenstein. En su lugar, Llano considera que el «modelo» fregeano constituye para la filosofía analítica actual, incluida Anscombe, el hilo conductor que permitiría establecer una continuidad entre las propuestas del primer y el segundo Wittgenstein, salvando a su vez las distintas paradojas que en cada caso se originan. A este respecto afirma Llano: «La vuelta a Frege [...] es una manifestación del señalado proceso autocrítico, del que resulta un nuevo clima filosófico. Todavía en 1959, podía decir Elizabeth Anscombe que la grandeza de Frege había sido reconocida por muy pocos, con las eminentes excepciones de Russell y Wittgenstein, (aunque carecieran de) una adecuada comprensión de su predecesor». ${ }^{20}$

Evidentemente la prolongación de Wittgenstein mediante la teoría del significado de Frege debe a su vez tratar de superar numerosas paradojas que ya habrían sido señaladas por Wittgenstein y por Apel. Especialmente la concepción del sujeto intérprete relacional en el Tractatus como un «yo metafísico» en sí mismo paradójico. En efecto, las propuestas de Frege volverían a producir una relación similar a la que el «ojo» mantiene con su respectivo «campo visual»,

17 Anscombe, M., Intentions, Ibidem, $95 \mathrm{p}$.

18 Llano, A.; Metafísica y lenguaje, Eunsa, Pamplona, 1983.

19 Apel, K. O., Transformación de la filosofía, T I-II, Suhrkamp, 1973; Taurus, Madrid, 1985.

20 Llano, A.; Metafisica y lenguaje, Ibidem, 54 p. 
como si el «ojo» pudiera relacionarse con la totalidad de los hechos empíricos que a su vez engloban el respectivo ámbito de la experiencia. Sin embargo ni el «ojo» ni el sujeto intérprete relacional o sus posibles interlocutores pueden observarse a sí mismos, ni pueden reducirse a un simple hecho empírico de su respectivo «campo de visión». De ahí que al final las propuestas del Tractatus dieran lugar a la aparición de una paradoja solipsista donde ni puede afirmarse la presencia del sujeto intérprete relacional, ni tampoco puede negarse. Por eso el primer Wittgenstein habría acabado optando por el más clamoroso de los silencios acerca de dicho «yo» metafísico relacional, a pesar de su necesaria mediación para el desarrollo del saber científico. En esa situación solo le queda postular una identidad entre mundo y vida. A este respecto afirma Llano, coincidiendo a este respecto con Apel, pero refiriéndose ahora también a Frege: «Se pregunta entonces Wittgenstein: «¿Dónde en el mundo puede observarse el sujeto metafísico? Tú dices, que aquí ocurre exactamente como con el ojo y el campo de visión; pero tú no ves realmente el ojo. Y nada en el campo de visión permite concluir que es visto por un ojo»». (Tractatus, \& 5.633). Como comenta Apel: «Con ello hemos descubierto la verdadera razón de por qué, para la filosofía transcendental del primer Wittgenstein, no puede darse ningún discurso con sentido del lenguaje sobre sí mismo y sobre su relación con el mundo ( Cf. Tractatus, \& 5.633)». (Y prosigue Llano). Wittgenstein lo dice por modo de aforismo: «Mundo y vida son lo mismo». ${ }^{21}$

En cualquier caso Llano considera que el segundo Wittgenstein en las Investigaciones no habría conseguido superar esta paradoja por el recurso a la máxima pragmática de Peirce, como pretende Apel. En su lugar Wittgenstein habría seguido más bien la teoría antipsicologista del significado de Frege, como también habría hecho notar Anscombe. En efecto, si el ojo no forma parte de un determinado campo de visión, se debe a que una cosa es el mirar un objeto a través de los sentidos y otra muy distinta su consideración con los ojos de la mente, al modo como sucede con el «yo metafísico» relacional, según el segundo Wittgenstein. En este sentido Wittgenstein habría establecido una clara separación entre las razones para actuar basadas en hechos que están a su vez dentro de un determinado campo de visión, y aquellas otras razones para creer que están basadas en simples opiniones, hábitos o conceptos que sólo se ven a través de los ojos de la mente. Y en este mismo sentido Frege habría establecido una clara separación entre los objetos que pueden ser vistos a través de los sentidos, y los conceptos u objetos indeterminados que, como la «ballena», solo pueden concebirse a través del propio entendimiento. A este respecto afirma Llano: «Para Frege, no hay ninguna entidad intermedia

21 Wittgenstein, L.; Tractatus, \& 5.631; Apel, K-O.; Transformacion de la Filosofia, I, Ibidem, 242 p.; Llano, A.; Metafisica y lenguaje, Ibidem, 39 p. 
entre conceptos y objetos. Como sostiene en Fundamentos de la Aritmética, «la palabra «ballena» no denomina ningún ser individual». ${ }^{22}$

A este respecto Llano reconstruye el importante papel que podría desempeñar la teoría del significado de Frege en la superación de la paradoja solipsista que se acabó haciendo presente en el Tractatus. Pero simultáneamente también se pretende hacer ver la importancia que tuvo la crítica al psicologismo de Frege en el rechazo del modo solipsista como la filosofía transcendental en general y el primer Wittgenstein en especial, habría seguido concibiendo al sujeto intérprete relacional de los signos lingüísticos y a sus posibles interlocutores. Frege habría comprobado como la universalidad que se predica de los conceptos no se origina específicamente en el alma humana a partir de la observación de la experiencia. En su lugar los conceptos se justifican en virtud de unas razones lógicas muy precisas, con independencia de que también deben ser confirmados mediante una segunda vuelta a la experiencia (o la llamada conversio ad phntasmata, por los clásicos). A este respecto afirma Llano: «La argumentación de Frege es aguda y vigorosa. Merece la pena que resuene con sus propias palabras: 'Hay que recordar, según parece, que las proposiciones no dejan de ser verdaderas cuando yo dejo de pensar en ellas; así como el sol no se aniquila cuando yo cierro los ojos. [...] Si en el flujo permanente todas las cosas no permaneciera nada firme, eterno, desaparecería la inteligibilidad del mundo y todo se precipitaría en la confusión. Algunos piensan [...] explicarlo psicológicamente a partir de la naturaleza del alma humana. Pero esta concepción aboca a lo subjetivo $y$, si se prosigue hasta el final, suprime la verdad'». ${ }^{23}$

En cualquier caso la aceptación de la crítica al psicologismo de Frege por parte de la filosofía analítica actual exigió retrotraer su fundamentación al Tractatus del primer Wittgenstein, a fin de evitar la ulterior aparición de este tipo de paradojas. De este modo se podrían reconstruir sus propuestas como un proyecto inevitablemente fallido. Sin embargo habría permitido a su vez justificar el paso dado hacia una visión más autocrítica de sus iniciales postulados heurísticos en las Investigaciones Filosóficas, una vez que también se habría producido un progresivo acercamiento al realismo semiótico defendido por Frege. Se pretende así reconstruir el efectivo tránsito que también se habría operado en Frege desde un sujeto intérprete relacional a otro de carácter estrictamente personal. En efecto, en la medida que el propio sujeto asume la responsabilidad de la justificación otorgada a los correspondientes conceptos, tampoco habría lugar para el tipo de paradojas y de lagunas anteriormente provocadas por el psicologismo, el positivismo y el subjetivismo de la filosofía de la conciencia presentes en Wittgenstein. A este respecto afirma Llano:

22 Llano, A.; Metafisica y lenguaje, Ibidem. 103 p.

23 Llano, A.; Metafísica y lenguaje, Ibidem, $53 \mathrm{p}$. 
«A través de la crítica al psicologismo, Frege no sólo se destaca netamente on respecto al positivismo y al subjetivismo de su entorno histórico, sino que propone «implícitamente» una alternativa a la filosofía de la conciencia». ${ }^{24}$

A este respecto Frege habría establecido el punto de partida de la crítica al psicologismo y al representacionismo en un lugar muy concreto: la estricta separación existente entre lo psicológico y lo lógico, entre lo subjetivo y lo objetivo, entre lo no demostrable y lo demostrable mediante la razón. Este habría sido el modo de explicar la diferencia entre el modo como el sujeto intérprete relacional percibe los objetos de la experiencia sensible y el modo de concebir los respectivos conceptos de la inteligencia o de la persona, sin establecer una simple correlación crítico-transcendental o pragmático transcendental entre ellos. No se pueden concebir el concepto como si se tratara de un simple conglomerado de sensaciones y de experiencia empíricas, por mucho que se compartan, cuando lo específico del concepto sería establecer una relación universal estrictamente lógica que ya propiamente no pertenece al ámbito de la sensación o de la experiencia empírica, ni se inserta en un determinado campo de visión de naturaleza crítico-transcendental. De ahí la estricta separación que Frege habría establecido entre los conceptos y los objetos, entre lo universal y lo singular, por opinar que en ningún caso los conceptos pueden reducirse a un simple conglomerado de objetos singulares, por muy similares que parezcan. El concepto viene definido más bien por la relación lógica universal que se establece entre un conjunto de individuos, que posteriormente puede ser confirmada en la experiencia, pero que como tal no se encuentra en ninguno de ellos. A este respecto afirma Llano, citando a Anscombe: «En la Introducción al mencionado libro (los Fundamentos de la Aritmética), Frege indica que, entre los principios fundamentales a los que se atendrá su investigación, el primero es este: «Hay que separar tajantemente lo psicológico de lo lógico, lo objetivo de lo subjetivo». ${ }^{25}$

A este respecto, Llano hace notar como también el primer Wittgenstein habría pretendido aplicar una estricta separación entre los conceptos y los objetos, al modo señalado por Frege. Sin embargo al final la actitud antimetafísica de Wittgenstein le habría impedido remitirse a ningún tipo de presupuesto previo inobservable que no estuviera basado directamente en la experiencia, viéndose obligado a optar por una fundamentación crítico-transcendental de las leyes lógicas y físico-matemáticas. De este modo la universalidad de los conceptos y leyes se tuvo que reducir a un mero conglomerado de hechos empíricos, como al final sucede en el psicologismo naturalista e idealista del Tractatus. En este contexto el papel aglutinador desempeñado por el sujeto intérprete relacional

24 Llano, A.; Metafísica y lenguaje, Ibidem, 51-52 pp.

25 Llano, A; Metafisica y lenguaje, Ibidem, 52 pp. 
y sus posibles interlocutores se acabó volviendo muy paradójico, al tenerse que apropiar de una universalidad lógica y matemática que ya no se podía reducir a una simple superposición crítico-transcendental de sensaciones fenoménicas. A este respecto afirma Llano: «El relativismo con el que se enfrenta Frege es el psicologismo naturalista e idealista: una suerte de reduccionismo, que intenta disolver la especificidad de las leyes lógicas y matemáticas, convirtiéndolas en leyes básicamente psicológicas». ${ }^{26}$

En cualquier caso en su segunda época Wittgenstein cambiaría radicalmente el modo de legitimar la actividad del sujeto intérprete relacional, pasando a tener un carácter cada vez más personal. Para Llano, el hilo conductor que permite establecer una continuidad entre las propuestas del primer y el segundo Wittgenstein se sitúa en la progresiva dependencia que a lo largo de ambos periodos habría mantenido con el «modelo» fregeano. De todos modos no deja de ser paradójico que este acercamiento a Frege fuera llevado a cabo por los filósofos que más lo desacreditaron, en referencia a Russell. De hecho le habrían acusado persistentemente de una forma de psicologismo y representacionismo similar al que el propio Frege había criticado y que estaba muy dejos de defender. De todos modos Llano opina que el segundo Wittgenstein inicia ya la vuelta al sujeto intérprete personal de Frege, al modo como posteriormente se acabaría generalizado en la mayor parte de la filosofía analítica. Solo así se puede justificar la actitud autocrítica que el segundo Wittgenstein mantiene respecto del solipsismo y el representacionismo de sus iniciales posiciones en el Tractatus, aunque las hubiera formulado de una forma paradójica. A este respecto afirma Llano: «Las investigaciones de Frege no fueron en absoluto psicológicas y rechazaba que los contenidos mentales privados tuvieran relevancia filosófica». ${ }^{27}$

En cualquier caso el segundo Wittgenstein habría tenido un progresivo acercamiento en el modo de concebir al sujeto intérprete personal y a sus posibles interlocutores. Eso explica, según Llano, que con posterioridad al segundo Wittgenstein se hubiera producido una vuelta generalizada a la noción de signo interpretante de Frege y a la necesidad de admitir determinados presupuestos sobreentendidos que se encuentran implícitos en el uso del lenguaje. Especialmente cuando el sujeto intérprete relacional del segundo Wittgenstein ya no pudo justificar los respectivos signos universales de un modo representacionistas. De este modo el lenguaje tampoco se pudo remitir a unos conceptos concebidos al modo de una simple imagen mental, como si se trataran de un simple cúmulo de experiencias subjetivas. En su lugar, los conceptos tuvieron que concebirse de un modo objetivo como un signo interpretante de los

26 Llano, A.; Metafisica y lenguaje, Ibidem, 53 p.

27 Llano, A.; Metafísica y lenguaje, Ibidem, 54 p.

Contrastes vol. XXIV-N² (2019) 
respectivos presupuestos sobreentendidos previos. En cualquier caso se hizo inevitable la referencia a la correspondiente noción de realidad metafísica, teniendo que admitir un definitivo tránsito desde un sujeto intérprete meramente relacional a otro de carácter estrictamente personal. A este respecto Llano afirma: «La clave de esta inflexión final [...] la hemos situado ya en la crítica al representacionismo, en el rechazo de la primacía epistemológica del concepto como imagen mental. A mi juicio, las raíces históricas de este rechazo hay que buscarlas en la crítica al psicologismo acometida por Gottlob Frege». ${ }^{28}$

En cualquier caso los conceptos no pueden prescindir del lenguaje, como la persona tampoco puede prescindir del mundo ni de sus respectivos interlocutores. En cambio en la filosofía pragmatista del lenguaje ocurre lo contrario, salvo excepciones. En efecto, hay autores donde se reconoce que sin conocimiento tampoco se puede legitimar el triple uso icónico, indexical y performativo de los actos de habla, como sucede en los casos de Kripke y Strawson. Pero, por lo general, el pragmatismo prescinde de la noción de signo formal, de modo que el sujeto intérprete relacional tampoco puede mantener una relación intencional respecto del mundo como totalidad, o con sus posibles interlocutores. Solo cabe que el sujeto intérprete relacional se reduzca a un todo indiferenciado donde la colectividad se sobrepone a la individualidad de la persona. Por ejemplo, «saber un idioma» se tiene que concebir como un simple signo instrumental cuyo uso requiere el concurso de una técnica puesta al servicio de las relaciones intersubjetivas, sin necesidad de remitirse de un modo directo a la realidad del mundo circundante. Pero incluso en ese caso se priva al uso instrumental del lenguaje de su sentido más auténtico reduciéndolo a una simple práctica psicológica. Se olvida así que el lenguaje es un instrumento al servicio de la razón y, en definitiva, de la persona. A este respecto afirma Llano: «La reposición de la teoría del concepto como signo formal da, por tanto, la clave para un adecuado planteamiento de este problema. El signo formal se contrapone al signo instrumental. [...] Y, en este sentido, es también muy cierto lo que sostiene Wittgenstein: «entender un lenguaje significa dominar una técnica» [...] Pero de lo dicho no se puede valer quien proponga una explicación meramente pragmática, [...] que ignora el hecho fundamental de que el lenguaje es un instrumento de la razón, la cual transciende los procesos causales del mundo». ${ }^{29}$

El lenguaje se impone así de un modo convencional arbitrario a través de un específico proceso semiótico de denominación y comprobación de sus respectivos objetos. En cambio, los conceptos se legitiman en virtud de unas

28 Llano, A.; Metafísica y lenguaje, Ibidem, $51 \mathrm{p}$.

29 Llano, A.; Metafisica y lenguaje, Ibidem, 74-75 pp.; Wittgenstein, L,; Investigaciones Filosóficas, Ibidem. 199\&, 381 p. 
relaciones lógicas de estricta universalidad que son descubiertas por la inteligencia y a su vez se pueden ver confirmadas en la experiencia. De todos modos los conceptos solo podrían haber alcanzado su auténtico carácter como signo formal debido a la apertura metafísica tan singular que pone de manifiesto la intimidad de la persona; es decir, una especial capacidad para establecer una recíproca articulación entre la triple dimensión epistémica, semiótica y vital otorgada a las formas y signos intencionales a la hora de remitirse a los objetos de un determinado mundo entorno. Solo así la persona se puede concebir como un signo formal conceptual que, por un lado, le permite estar permanentemente abierta al mundo, pero que, por otro lado, es capaz de identificar su propia intimidad al reconocer esta capacidad que tiene de elaborar conceptos. A este respecto afirma Llano: «Recurramos a las viejas definiciones: «Signo formal es el que, sin previa noticia de sí mismo, súbita e inmediatamente representa algo distinto de sí. [...] El lenguaje - como signo instrumental - impone su presencia. El pensamiento, en cambio, [...] es operación inmanente, no movimiento. (....) Lo cual se manifiesta lingüísticamente en que las operaciones del pensamiento se pueden designar igualmente con el presente y con el pretérito perfecto del verbo correspondiente, porque «uno piensa y ha pensado, entiende $y$ ha entendido»». ${ }^{30}$

En Conclusión: Llano comprueba la estrecha correlación que la actual filosofía analítica más creativa establece entre la persona, el lenguaje y la apertura al mundo de la metafísica. En este contexto se concibe al lenguaje como la más elevada actividad inmanente de la persona humana capaz de expresar las virtualidades tan diversas de su propia intimidad. De este modo se asigna a la persona la poiesis o forma de acción primordial, mediante la que resulta posible dotar de un sentido intencional a los propios pensamientos a fin de remitirlos a un mundo concebido como totalidad. Sólo así la persona puede manifestar de un modo objetivo el «ser» de las cosas y a la vez el de su intimidad más auténtica. En este contexto la correlación que se establece entre el lenguaje, el pensamiento y la conducta no es una simple relación sobrevenida de un modo convencional y arbitrario. Se trata más bien de una conexión intrínseca con la pretensión de poder dotarse de sentido. En efecto, en la misma medida que la persona pone a prueba de una forma objetiva sus propias creaciones conceptuales y lingüísticas, en esa misma medida es capaz de legitimarlas como verdaderas o como falsas. De este modo la persona se afirma un signo intérprete de carácter formal, al modo señalado por Frege y por toda la tradición metafísica. A este respecto afirma Llano. «El lenguaje es la poiesis primordial. [...] La estructura lógica incorporada al lenguaje no

30 Cf. Aristóteles, Metaph. IX, 7; Llano, A.; Metafisica y lenguaje, Ibidem, 76-77 pp.). 
es una evidencia del pensar: es el pensar; y su relación con el lenguaje es de causalidad formal»..31

\section{CONCLUSIÓN:}

¿CABe un discurso interpersonal después de Wittgenstein y Apel?

Las propuestas de Apel prolongaron los planteamientos del último Wittgenstein, tratando de evitar a su vez las paradojas que originó su noción de sujeto intérprete, aunque sin conseguirlo. En la práctica se generaron otras aún más persistentes, que a su vez exigieron otras propuestas de prolongación por parte de Anscombe y Llano. En efecto, en vez de recurrir a Peirce, se remitieron a la teoría del discurso en Aristóteles, o retomaron con nuevo vigor las propuestas de Frege. En cualquier caso el reconocimiento del ser humano como persona permitió legitimar un discurso racional entre los distintos interlocutores interpelados, así como la ulterior fijación de unas condiciones éticas de sentido, que a su vez están sobreentendidas tras la transformación conceptual acaecida en el modo de fundamentar la propia metafísica.

Carlos Ortiz de Landázuri es profesor asociado de la Universidad de Navarra desde el curso 1976/77. Desde hace veinte años dirige la sección de "Novedades Bibliográficas de la revista Anuario Filosófico.

Lineas de investigación

Metodología y teoría de la ciencia, especialmente en Wittgenstein, Heidegger, Popper, Gadamer, Gombrich, Anscombe, von Wright, Apel, Habermas, la postmodernidad, la teoría del arte, la filosofía de las matemáticas y de la lógica.

Email: cortiz@unav.es

31 Llano, A.; Metafisica y lenguaje, Ibidem. 83 p. 
